International Journal of Pure and Applied Mathematics

Volume 90 No. 4 2014, 465-469

ISSN: 1311-8080 (printed version); ISSN: 1314-3395 (on-line version)

url: http://www.ijpam.eu

doi: http://dx.doi.org/10.12732/ijpam.v90i4.8

ijpam.eu

\title{
QUASI-PERIODIC SOLUTIONS OF EINSTEIN-FRIEDMAN EQUATIONS
}

\author{
Sergey V. Ershkov \\ Institute for Time Nature Explorations \\ M.V. Lomonosov's Moscow State University \\ Leninskie Gory, 1-12, Moscow, 119991, RUSSIA
}

\begin{abstract}
A new quasi-periodic solutions of Einstein-Friedman equations are presented here.

The equation for law of energy saving is proved to be transformed to the proper Abel ordinary differential equation.

The equation for evolution of the density of inter-stellar matter is reduced to linear ODE in the case of arbitrary equation of state. The equation of state in a form of linear connexion between the density and pressure of inter-stellar matter is used for the obtaining of such a solution. Besides, the component of solution for the density of inter-stellar matter is proved to be expressed in term of a proper quasi-elliptical integral.

The component of solution for the radius of space curvature is expressed depending on the density of inter-stellar matter.
\end{abstract}

AMS Subject Classification: 83CXX, 83F05

Key Words: Einstein-Friedman eqs., radius of space curvature, Abel ODE

\section{Introduction, the System of Equations}

The Einstein-Friedmann equations are a set of equations in physical cosmology

Received: October 10, 2013

(c) 2014 Academic Publications, Ltd. url: www.acadpubl.eu 
that govern the expansion of space in homogeneous and isotropic models of the universe within the context of general relativity. They were first derived by Alexander Friedmann in 1922 from Einstein's field equations of gravitation for the Friedmann-Lemaître-Robertson-Walker metric and a fluid with a given mass density $\rho$ and pressure $P$. The equations for negative spatial curvature were given by Friedmann in 1924, see [1].

In accordance with [1], the Einstein-Friedman system of equations should be presented as below:

$$
\begin{aligned}
k \cdot(c / R)^{2}+\left(R^{\prime} / R\right)^{2}+2\left(R^{\prime \prime} / R\right) & =-8 \pi G \cdot P / c^{2}, \\
k \cdot(c / R)^{2}+\left(R^{\prime} / R\right)^{2} & =8 \pi G \cdot \rho / 3 .
\end{aligned}
$$

Here $G$ is the gravitational constant, $c$ is the velocity of light; $P$ and $\rho$ are the pressure and the density of inter-stellar matter; $k=0,1$ or -1 in dependence on the sign of the curvature of space.

\section{Exact Solution}

Let us represent (1.2), which is the equation for law of energy saving, in other form:

$$
\left(R^{\prime}\right)^{2}-(8 \pi G / 3) \cdot \rho(t) \cdot R^{2}+k \cdot c^{2}=0 .
$$

If for the solutions $R(t)$ of such an equation we assume $\lambda(t)=R(t) / R^{\prime}(t)$, then we could obtain $(k \neq 0)$ :

$$
R^{\prime}=\left(\frac{-k \cdot c^{2}}{1-(8 \pi G / 3) \cdot \rho(t) \cdot \lambda^{2}}\right)^{1 / 2}, \quad R=\lambda \cdot\left(\frac{-k \cdot c^{2}}{1-(8 \pi G / 3) \cdot \rho(t) \cdot \lambda^{2}}\right)^{1 / 2}
$$

The right part of the 1-st of above equalities is proved to be a proper differential of the right part of 2-nd equality; such a fact let us obtain the proper Abel equation (see [2]):

$$
\lambda^{\prime}=C_{0} \cdot \rho^{\prime}(t) \cdot \lambda^{3}+2 C_{0} \cdot \rho(t) \cdot \lambda^{2}+1, \quad C_{0}=-(4 \pi G / 3) .
$$

We should note that Abel equation (2.1) does not depend on the sign of $k$. The linear combining of equation (1.1) and (1.2) letting us to

$$
R^{\prime \prime} / R=C_{0} \cdot\left\{\rho+\left(3 P / c^{2}\right)\right\} .
$$


Differentiation of equation (1.2) with respect to $t$ yields (see [3]):

$$
R^{\prime \prime} / R=-C_{0} \cdot\left\{\left(R / R^{\prime}\right) \cdot \rho^{\prime}+2 \rho(t)\right\},
$$

then eliminating equation (2.2) with equation (2.3), one obtains

$$
R / R^{\prime}=\lambda=-3\left(\frac{\rho+\left(P / c^{2}\right)}{\rho^{\prime}}\right)
$$

Thus, substituting of expression (2.4) to equation (2.1) yields

$$
\begin{aligned}
& \left(-3\left(\frac{\rho+\left(P / c^{2}\right)}{\rho^{\prime}}\right)\right)^{\prime}=C_{0} \cdot \rho^{\prime}(t) \cdot\left(-3\left(\frac{\rho+\left(P / c^{2}\right)}{\rho^{\prime}}\right)\right)^{3} \\
& +2 C_{0} \cdot \rho(t) \cdot\left(-3\left(\frac{\rho+\left(P / c^{2}\right)}{\rho^{\prime}}\right)\right)^{2}+1, \\
& \Rightarrow-3\left(\frac{\left(\rho^{\prime}+\left(P^{\prime} / c^{2}\right)\right) \cdot \rho^{\prime}-\rho^{\prime \prime} \cdot\left(\rho+\left(P / c^{2}\right)\right)}{\left(\rho^{\prime}\right)^{2}}\right) \\
& =C_{0} \cdot \rho^{\prime}(t) \cdot\left(-3\left(\frac{\rho+\left(P / c^{2}\right)}{\rho^{\prime}}\right)\right)^{3}+2 C_{0} \cdot \rho(t) \cdot\left(-3\left(\frac{\rho+\left(P / c^{2}\right)}{\rho^{\prime}}\right)\right)^{2}+1 \text {, }
\end{aligned}
$$

or (let $c=1$, for simplicity):

$$
\rho^{\prime \prime} \cdot(\rho+P)=-3 C_{0} \cdot(\rho+P)^{2} \cdot\{\rho+3 P\}+\rho^{\prime} \cdot\left(\frac{4}{3} \rho^{\prime}+P^{\prime}\right) .
$$

In either case, however, one needs another independent equation to solve for $\rho(t)$. This is usually given by an equation of state of the form $P=P(\rho)$, see $[3]$.

So, the proper changing of variables: $y(\rho)=\rho^{\prime}(t)$ let us rewrite equation (2.5) to the linear ODE below:

$$
\begin{gathered}
y \cdot y^{\prime} \cdot(\rho+P(\rho))=-3 C_{0} \cdot(\rho+P(\rho))^{2} \cdot\{\rho+3 P(\rho)\}+\frac{4}{3} y^{2}+y \cdot P^{\prime}(\rho) \cdot y \\
\Rightarrow\left(y^{2}\right)^{\prime}=-6(\rho+P(\rho)) \cdot\{\rho+3 P(\rho)\} C_{0}+2 y^{2} \cdot\left(\frac{P^{\prime}(\rho)+\frac{4}{3}}{\rho+P(\rho)}\right) \cdot
\end{gathered}
$$




\section{Exact solution, the case $p=\omega \cdot \rho$}

In cosmology, the following simple relation is assumed: $P=\omega \cdot \rho, \omega=$ const. While the value of $\omega$ may in principle change with redshift, it is often assumed that $\omega$ is independent of time just for simplicity, see [3]. Then substituting this equation of state into the last equation (2.6) immediately yields ( $A=\mathrm{const}$; $\omega \neq-1, \omega \neq-1 / 3)$ :

$$
\begin{array}{r}
\left(y^{2}\right)^{\prime}=-6(\omega+1) \cdot(3 \omega+1) \cdot C_{0} \cdot \rho^{2}+\left(\frac{2}{\rho}\right)\left(\frac{\omega+(4 / 3)}{\omega+1}\right) \cdot y^{2} \\
\Rightarrow y=\frac{d \rho}{d t}= \pm \frac{\sqrt{A-\frac{6(\omega+1) \cdot(3 \omega+1) \cdot C_{0}}{\left(3-\frac{2 \omega+(8 / 3)}{\omega+1}\right)} \cdot \rho^{\left\{3-\frac{2 \omega+(8 / 3)}{\omega+1}\right\}}}}{\rho^{-\left(\frac{\omega+(4 / 3)}{\omega+1}\right)}}
\end{array}
$$

which could be transformed to the proper quasi-elliptical integral (see [4]) in regard to $\rho$.

Let us represent equation (2.4) in the form below $(P=\omega \cdot \rho, \omega=$ const; $c=1$, for simplicity):

$$
R^{\prime} / R=-\frac{3 \rho^{\prime} / \rho}{\omega+1} \Rightarrow R(t)=R(0) \cdot\left(\frac{\rho(t)}{\rho(0)}\right)^{-\frac{3}{\omega+1}}
$$

\section{Discussions}

The evolution of the universe as predicted by the Einstein-Friedman equations [5] when dominated by a single, isotropic, stable, static, perfect-fluid energy form is considered for different values of the gravitational pressure to density ratio $\omega$.

These energy forms include phantom energy $(\omega<-1)$, cosmological constant $(\omega=-1)$, domain walls $(\omega=-2 / 3)$, cosmic strings $(\omega=-1 / 3)$, normal matter $(\omega=0)$, radiation and relativistic matter $(\omega=1 / 3)$, and a previously little-discussed form of energy called "ultralight" $(\omega>1 / 3)$, see [5]. The main result, which should be outlined, is that the dependence of density of inter-stellar matter in expanding Universe is proved to be given by a proper quasi-elliptical integral. But the elliptical integral is known to be a generalization of a class of inverse periodic functions. Thus, by a proper obtaining of re-inverse dependence of a solution from time-parameter we could present the entire evolution of Universe as a set of quasi-periodic cycles. 


\section{Conclusion}

Thus, we have obtained absolutely new quasi-periodic solution of EinsteinFriedman equations (1.1)-(1.2). The equation for law of energy saving (1.2) is proved to be transformed to the proper Abel ordinary differential equation (2.1). The equation for the density of inter-stellar matter is reduced to linear ODE (2.6) in the case of arbitrary equation of state $P=P(\rho)$. Also the equation of state in a linear form $P=\omega \cdot \rho, \omega=$ const is considered. In such a case, the component of solution for the density of inter-stellar matter is proved to be expressed in term of a proper quasi-elliptical integral (3.1). The component of solution for the radius of space curvature is expressed depending on the density of inter-stellar matter (3.2).

\section{References}

[1] A.A. Friedmann, Über die Möglichkeit einer Welt mit konstanter negativer Krümmung des Raumes, Z. Phys., 21, No. 1 (1924), 326-332.

[2] E. Kamke, Hand-Book for Ordinary Differential Equations, Moscow, Science, 1971.

[3] O. Lahav, Y. Suto, Measuring our universe from galaxy redshift surveys, Living Rev. Relativity, 7 (2004), 8.

[4] D. Lawden, Elliptic Functions and Applications, Springer-Verlag, 1989.

[5] R.J. Nemiroff, B. Patla, Adventures in Friedmann cosmology: A detailed expansion of the cosmological Friedmann equations, American Journal of Physics, 76, No. 3 (2010), 265-276. 
